\title{
Evaluation of SARS-CoV-2 vaccination rate among healthcare workers in a tertiary hospital in Mogadishu Somalia: A cross-sectional study
}

Mohamed Osman Omar Jeele ( $\sim$ drjeele@gmail.com )

Mogadishu Somali Turkish Training and Research Hospital

Ahmed Muhammad Bashir

Mogadishu Somali Turkish Training and Research Hospital

Liban Hassan Jimale

Mogadishu Somali Turkish Training and Research Hospital

Abdulkamil Abdullahi Adani

Mogadishu Somali Turkish Training and Research Hospital

\section{Research Article}

Keywords: SARS-CoV-2 infection, SARS-CoV-2 vaccination rate, COVID-19, Healthcare workers, Somalia

Posted Date: November 30th, 2021

DOI: https://doi.org/10.21203/rs.3.rs-1077568/v1

License: (c) (i) This work is licensed under a Creative Commons Attribution 4.0 International License.

Read Full License 


\section{Abstract \\ Background}

The percentage of healthcare workers that are vaccinated against SARS-CoV-2 in Somalia remains unknown. The main purpose of our study is to determine the SARS-CoV-2 vaccination rate among healthcare workers in a tertiary hospital in Mogadishu, Somalia.

\section{Methods}

The design of the study is cross-sectional which was undertaken to assess the vaccination rate of healthcare workers in Mogadishu Somali Turkish Training and Research Hospital from September 1 to September 20, 2021. A total number of 210 healthcare workers have participated in this study. The data were collected using a questionnaire.

\section{Results}

Out of 210 healthcare workers that have enrolled in the study $56.2 \%(n=118)$ were males. The mean age of the participants was 28.23 with SD \pm 2.9 . The most abundant age group in our study was $18-27$ years with $53.8 \%(n=113)$. According to the participant's profession, the doctors were the commonest participant with $48.6 \%(n=102)$. The SARS-CoV-2 vaccination level of the healthcare workers in Mogadishu Somali Turkish Training and Research Hospital was found to be $20 \%(n=42) .26 .2 \%$ of the males that participated in this study were vaccinated against SARS-CoV-2 while only $12 \%$ of females were vaccinated. The study found that there is a significant correlation between SARS-CoV-2 vaccination and gender with $p$ value $<.05$. The study also revealed that there is a negative correlation between age and being vaccination against Covid-19 disease with $p$ value $<.01$. 30\% $(n=31)$ of the doctors, $5.6 \%(n=4)$ of nurses, $14.2 \%(n=14)$ of radiology technicians, $30.7 \%(n=4)$ of anesthesia technicians, and $10 \%(n=1)$ of laboratory stuffs were vaccinated against SARS-CoV-2. The reasons for SARS-CoV-2 vaccine hesitancy were fear towards the vaccine complications $58 \%(n=97)$, followed by believed that vaccine will not be beneficial, lack of opportunity, no reason, and avoiding for religious or social reasons in $23 \%(n=39), 8.3 \%$ $(n=14), 9 \%(n=15), 1.7 \%(n=3)$, respectively.

\section{Conclusion}

We found that only $20 \%$ of the healthcare workers in a tertiary hospital in Somalia were vaccinated against SARS-CoV-2 infection. This is an emergency public health situation as it will put the healthcare workers and the patients at risk for developing Covid 19 disease.

\section{Background}


SARS-CoV-2 infection not only became a major global health burden when it's first discovered in late 2019 , but it also became an economic and social burden throughout the world ${ }^{(1)}$. In many countries, Covid 19 vaccine was approved for the general population at the end of 2020 and early $2021^{(2,3)}$. SARSCoV-2 vaccines were evaluated through many clinical trials and demonstrated to have a high level of protection against the Covid-19 disease ${ }^{(1)}$.

In accordance with their profession, healthcare workers (HCW) are one of the most prone population groups to contract Covid-19 disease ${ }^{(4)}$. Due to that reason, HCW was identified as one of the priority groups to receive SARS-Cov-2 vaccine worldwide ${ }^{(5)}$. Despite these efforts from the governments and institutions to vaccinate $\mathrm{HCW}$, the acceptance rate of vaccination among HCW is low. For instance, a poll that was arranged by the Kaiser family foundation in December 2020 found that $29 \%$ of the HCWs were hesitant to get COVID-19 vaccines where $27 \%$ of the individuals in the general population did not want to take the vaccine ${ }^{(3,6)}$.

Biswas et al. found that more than 75,000 healthcare professionals around the world estimated that more than a fifth of the healthcare workers globally was not willing or hesitant to take SARS-Cov-2 vaccinations in $2021^{(3,7)}$.

Meanwhile, the Somali ministry of health reported its first confirmed SARS-Cov-2 infection in mid-March 2020. As of this date, the country reported a total of 22,369 cases with 1,238 death cases ${ }^{(8)}$.

In early March 2021, the first doses of SARS-CoV-2 vaccine arrived in Somalia through COVAX facility. Therefore, the Somali government rushed to vaccinate frontline HCW and the people with the highest risk

(9). The government has distributed at least 668,104 SARS-CoV-2 vaccine shots at the time of writing this article $^{(10)}$.

To the extent of our knowledge, the percentage of HCW that is vaccinated against SARS-CoV-2 infection in Somalia remains unknown. The main purpose of our study is to determine SARS-CoV-2 vaccination rate among healthcare workers in a tertiary hospital in Mogadishu, Somalia. This study also intends to investigate the reasons HCW in Somalia are hesitant to take SARS-CoV-2 vaccine.

\section{Methods}

The main goal of our study is to determine the SARS-CoV-2 vaccination rate among healthcare workers in a tertiary hospital in Mogadishu, Somalia.

\section{Study Population, Design, and Data Collection:}

This study is a single center-based study done in Mogadishu Somali Turkish Training and Research Hospital. The hospital is a tertiary hospital that has all the health departments and serves at least one thousand patients per day. There are more than 500 healthcare workers who are actively working in the 
hospital. A total number of 210 healthcare workers from Mogadishu Somali Turkish Training and Research Hospital have agreed and gave their consent to participate in the study.

The SARS-CoV-2 vaccines that Mogadishu Somali Turkish Training and Research Hospital healthcare workers received were Oxford AstraZeneca vaccine and Sinopharm COVID-19 vaccine.

The design of the study is a cross-sectional study that was undertaken to assess the vaccination rate of healthcare workers in Mogadishu Somali Turkish Training and Research Hospital from September 1 to September 20, 2021.

We collected the data using a questionnaire which was prepared in a google form document. A link to the google form questionnaire document was sent to every healthcare worker who works in Mogadishu Somali Turkish Training and Research Hospital through WhatsApp application. Some healthcare workers were not reached through WhatsApp application; thus, a written form of the same questionnaire was collected through a face-to-face interview by one of our authors to ensure the accuracy of the data.

The questionnaire was composed of 3 parts of questions. The first part of the questionnaire addressed the demographic profiles of the participants. The demographics that were obtained from this questionnaire included age, sex, and the profession of the participants. If the participant defines their profession as a doctor, there was a following question that asks the participant his or her department. The second part of the questionnaire composed of questions about the level of SARS-CoV- 2 vaccination of the participant. It also encompasses questions for the reasons for not getting the SARS-CoV-2 vaccine if the respondent is not vaccinated. The last part of the questionnaire consisted of questions that only the vaccinated participants could answer. It contains questions that addressed if the vaccinated participant had encountered any complications after they received SARS-CoV-2 vaccine regardless of the type of the vaccine they have taken.

\section{Exclusion Criteria:}

All non-healthcare workers of Mogadishu Somali Turkish Training and Research Hospital were excluded from the study. Also, healthcare workers who refused to participate were excluded from this study.

\section{Statistical analysis}

The data that was collected through google form and the written questionnaires were then transferred to Microsoft Excel sheet and then imported to Statistical Package for Social Sciences (SPSS) software version 26. The data was finally analyzed using Statistical Package for Social Sciences (SPSS) software version 26. Descriptive statistics, compare means were mainly used in the study. Pearson's correlation was also used in this study. The analyzed data was presented as frequencies and percentages.

\section{Results}




\section{Demographics:}

Out of 210 healthcare workers that have enrolled in the study, $56.2 \%(n=118)$ were males and $43.8(n=92)$ were females. The mean age of the participants was 28.23 with SD \pm 2.9 (Table1). The age of the participants was further divided into age groups. The most abundant age group in our study was 18-27 years with $53.8 \%(n=113)$. The second most common age group in the study was $28-35$ years with $42.9 \%$ $(n=90)$, followed by $36-50$ years with $3.3 \%(n=7)$ (Table 2$)$.

According to the participant's profession, the doctors were the commonest participant with $48.6 \%$ $(n=102)$. The second most common participant were nurses with $33.8 \%(n=71)$, followed by radiology technicians, anesthesia technicians, and laboratory staffs with $6.7 \%(n=14), 6.2 \%(n=13), 4.8 \%(n=10)$, respectively (Table 2).

\section{SARS-CoV-2 vaccination level of the participants:}

The SARS-CoV-2 vaccination level of the healthcare workers in Mogadishu Somali Turkish Training and Research Hospital was found to be $20 \%(n=42)$. The remaining $80 \%$ of the participants $(n=168)$ were not vaccinated against SARS-CoV-2. $26.2 \%$ of the males that were participated in this study were vaccinated against SARS-CoV-2 whereas $12 \%$ of the females were vaccinated against SARS-CoV-2 (Table 2).

This study found that there is a significant correlation between SARS-CoV-2 vaccination and gender with $p$ value $<.05$. Also, the study revealed that there is a negative correlation between age and being vaccination against Covid-19 disease with $p$ value $<.01$ (Table 5).

According to participant's profession, $30 \%(n=31)$ of the doctors were vaccinated against SARS-CoV-2. This study also found that only $5.6 \%(n=4)$ of nurses were vaccinated against SARS-CoV-2. In addition, $14.2 \%(n=14)$ of radiology technicians were vaccinated against SARS-CoV-2, 30.7\% $(n=4)$ of anesthesia technicians, and $10 \%(n=1)$ of laboratory staffs were vaccinated against SARS-CoV-2. This study found no correlation between SARS-CoV-2 vaccination and the profession of the participants with $p$ value $=.18$ (Table 2).

\section{Reasons for not getting SARS-CoV-2 vaccination among the participants:}

Out of 210 participants, $80 \%$ ( $n=168)$ were not vaccinated against SARS-CoV-2. Of the 168 participants who were not vaccinated against SARS-CoV-2, the most common reason for not getting the SARS-CoV-2 vaccine was fear of the vaccine complications with $58 \%(n=97) .23 \%(n=39)$ of the participants believed that the vaccine will not be beneficial to them. $8.3 \%(n=14)$ said that they did not get an opportunity to get vaccinated. In addition, another $9 \%(n=15)$ responded that they did not get the vaccine for no reason, while the remaining $1.7 \%(n=3)$ responded to have avoided the SARS-CoV-2 vaccine for religious or social reasons (Table 3$)$. 


\section{Common side effects of SARS-CoV-2 vaccinations among the participants:}

Out of the 42 participants that have received SARS-CoV-2 vaccination, $42.8 \%(n=18)$ have reported side effects due to the vaccine regardless of the type of SARS-CoV-2 vaccine which they have received. Fever was the most frequented side effect that the participants reported with $78 \%(n=14)$. Body ache was the second most reported side effect due to SARS-CoV-2 vaccination with $72.2 \%(n=13)$, followed by pain at the site of injection with $44.4 \%(n=8)$. Our participants did not report any form of thrombosis or other serious condition as a complication due to SARS-CoV-2 (Table 4).

\section{Discussion}

The novel coronavirus infection 2019 (COVID-19) has been ongoing and infecting numerous people affecting their daily life in the process ${ }^{(11,12)}$. Authorities around the world have made multiple public health measures to halt the ongoing virus such as physical distancing, wearing masks, and a total lockdown which brought the world economy on a brink of collapse ${ }^{(11)}$.

Hence, this situation sparked the most extensive vaccine hunt in the history of medicine ${ }^{(13,14)}$. Many studies about SARS-CoV-2 vaccine have been done around the globe to know their safety and side effects on the population. A study done in Canada in 2021 by Vilches et al. suggested that SARS-CoV-2 vaccination could potentially decrease the number of people that could be infected by the virus and thus decreases the morbidity and mortality of the SARS-CoV-2 infection in the population ${ }^{(11)}$.

Some regions around the world have high acceptability rate of SARS-CoV-2 vaccination than other regions. For instance, in Vietnam, a study done by Huynh et al. in 2021 about the willingness of healthcare workers to get SARS-CoV-2 concluded that $76 \%$ of HCW were willing to get vaccinated for SARS-CoV-2 infection ${ }^{(15)}$. Another example is a cross-sectional study done in France by Gagneux-Brunon et al. in 2021 which assessed the intentions of HCW to get vaccinated against SARS-CoV-2 infection found that the intention to get the vaccine be $75 \%(16)$.

We conduct our study to get a clue about the level of vaccination among frontline health workers in Somalia. In the presenting study, we found that the level of SARS-CoV-2 vaccination rate among healthcare workers in Mogadishu Somali Turkish Training and Research Hospital to be $20 \%$. We found that $80 \%$ of healthcare workers were not vaccinated against SARS-CoV- 2 infection.

Furthermore, our study suggested that sex is a significant positive predictor to get SARS-CoV-2 vaccine as males were more vaccinated than females. This fact can be debatable since males were the common gender among the participants. This positive prediction between gender and vaccine was also backed by a metanalysis done by Biswas et al. in 2021 who also found that the male gender was more likely to accept SARS-CoV-2 vaccination than females ${ }^{(3)}$. 
Our study also found that $30 \%$ of the doctors who are working in Mogadishu Somali Turkish Training and Research Hospital were vaccinated. The SARS-CoV-2 vaccination level was much lower in nurses with only $5.6 \%$ of the nurses being vaccinated against SARS-CoV- 2 infection. This reality puts the nurses in great danger as they are the ones who contact the patients more often. Our finding also indicates that younger participants tend to be more likely vaccinated against SARS-CoV-2 than the older participants.

We did not find any association between the participant's profession and being vaccinated against SARSCoV-2.

Since most of our participants were not vaccinated against SARS-CoV-2 infection. The most common reason for not getting vaccinated among our participants was fear of the complications of the SARS-CoV2 vaccines. Also, a handful of participants believed that the vaccine will not help them or protect them from SARS-CoV-2 infection. That number was as high as $23 \%$. This was also a finding in a review metanalysis by Biswas et al. in 2021 who also found that the most common reasons for HCW hesitancy to be vaccinated against SARS-CoV-2 be vaccine safety, efficacy, and side effects ${ }^{(3)}$.

A cross-sectional study done by online by Mohamed al et al. in 2020 about SARS-CoV-2 vaccination acceptance in population living in Mogadishu found that $63.2 \%$ of the participants refused to take the vaccination if available ${ }^{(17)}$. Another cross-sectional study done through online by Ahmed et al. found that $76.8 \%$ of the respondents were willing to receive vaccine ${ }^{(18)}$.

In the presented study, we also asked our participants who were vaccinated against SARS-CoV-2 infection if they had experienced any complications after they get vaccinated regardless of the type of vaccines. Most of our participants said that they have experienced fever after they get the SARS-CoV-2 vaccination followed by body ache and pain at the site of injection. This cannot be used as future evidence for side effects of individual vaccine could have, so another wide range study is needed to determine the side effects of SARS-CoV-2 vaccination.

There are some limitations of our study. Those include a small sample size with only a single hospital healthcare workers. Nevertheless, we believe that our study can be representative of the SARS-CoV-2 vaccination level of the healthcare workers in Mogadishu as this is a tertiary hospital and the biggest care provider hospital in Mogadishu, Somalia.

\section{Conclusion}

In the presenting study, we found that only $20 \%$ of the healthcare workers in a tertiary hospital in Somalia were vaccinated against SARS-CoV-2 infection. Most of the respondents were hesitant to be vaccinated despite the availability of the vaccine. This is an emergency public health situation as it will put the healthcare workers and the patients at risk for developing Covid 19 disease. The policymakers must implement a new and effective strategy to raise the awareness of healthcare workers towards SARS-CoV2 vaccination. 


\section{Abbreviations}

COVID-19= coronavirus disease 19

$\mathrm{HCW}=$ healthcare workers

$\mathrm{n}=$ sample size

SARS-CoV-2 = severe acute respiratory syndrome corona virus 2

SPSS: Statistical Package for Social Sciences.

\section{Declarations}

We perform our study in accordance to the declaration of Helsinki.

\section{Competing of interest:}

The authors declare that they have no competing of interest.

\section{Funding source:}

The authors declare they have no funding source for this research.

\section{Acknowledgment:}

We thank to all participants who gave their consent to be involved in this study.

\section{Data Availability:}

The data is available from the corresponding author and can be accessed upon reasonable requested.

\section{Ethical consideration:}

Informed consent was obtained from every healthcare worker that participated in this study. This study does not reveal any personal data and the privacy of the participants was well protected. This study had been evaluated and approved by Mogadishu Somali Turkish Training and Research Hospital ethical committee.

\section{Authors contribution:}

Jeele, MOO, brought the concept of the study, collected the questionaires, perfomed data analysis, and prepared the manuscript.

Bashir, AM, participated the data collection. 
Jimale, LH, participated the manuscript preparation.

Adani, AA participated the manuscript preparation.

All authors read and approved the final manuscript before submitting.

\section{Consent for publication}

Not Applicable

\section{References}

1. Moghadas SM, Vilches TN, Zhang K, Nourbakhsh S, Sah P, Fitzpatrick MC, Galvani AP. Evaluation of COVID-19 vaccination strategies with a delayed second dose. PLoS biology. 2021 Apr 21;19(4):e3001211.

2. Anyiam-Osigwe T. COVID-19 vaccines are now approved in some countries. What will it take to approve them for the rest of the world. 2021.

3. Biswas N, Mustapha T, Khubchandani J, Price JH. The Nature and Extent of COVID-19 Vaccination Hesitancy in Healthcare Workers. Journal of Community Health. 2021 Apr 20:1-8.

4. La Colla L, Polidori MC, Di Saverio G, Preziosi G, Mantovani L, Caobelli F. The impact of health worker gap in Italy on the COVID-19 pandemic. A good time to improve the quality of the Italian National Health System (NHS)?. Journal of Infection and Public Health. 2020 Sep;13(9):1253.

5. Huang P. First COVID-19 Vaccine Doses to Go to Health Workers, Say CDC Advisers. Shots- Health News from NPR 2020; https://www.npr.org/sections/healthshots/2020/11/05/931844298/firstcovid-19-vaccine-doses-to-go-to-health-workers-saycdc-advisers.

6. hamel, L., et al. (2020). KFF COVID-19 vaccine monitor: December 2020. Retrieved from https://www.kf.org/coronaviruscovid-19/report/kf-covid-19-vaccine-monitor-december-2020/

7. Mustapha T, Khubchandani J, Biswas N. COVID-19 vaccination hesitancy in students and trainees of healthcare professions: A global assessment and call for action. Brain, Behavior, \& Immunity-Health. 2021 Jun 30.

8. https://covid19som-ochasom.hub.arcgis.com/

9. https://.

10. https://graphics.reuters.com/world-coronavirus-tracker-and-maps/countries-and-territories/somalia/

11. Vilches TN, Zhang K, Van Exan R, Langley JM, Moghadas SM. Projecting the impact of a two-dose COVID-19 vaccination campaign in Ontario, Canada. Vaccine. 2021 Apr 22;39(17):2360-5.

12. COVID, Coronavirus. "Global Cases [Internet]. Johns Hopkins Center for Systems Science and Engineering." (2020).

13. Voysey M, Clemens SA, Madhi SA, Weckx LY, Folegatti PM, Aley PK, Angus B, Baillie VL, Barnabas SL, Bhorat QE, Bibi S. Single-dose administration and the influence of the timing of the booster dose on 
immunogenicity and efficacy of ChAdOx1 nCoV-19 (AZD1222) vaccine: a pooled analysis of four randomised trials. The Lancet. 2021 Mar 6;397(10277):881-91.

14. WHO. The COVID-19 candidate vaccine landscape. Feb 16, 2021. https://www.who.int/publications/m/item/draft-landscape-of-covid19-candidate-vaccines (accessed Feb 17, 2021).

15. Huynh G, Tran TT, Nguyen HT, Pham LA. COVID-19 vaccination intention among healthcare workers in Vietnam. Asian Pacific Journal of Tropical Medicine. 2021 Apr 1;14(4):159.

16. Gagneux-Brunon A, Detoc M, Bruel S, Tardy B, Rozaire O, Frappe P, Botelho-Nevers E. Intention to get vaccinations against COVID-19 in French healthcare workers during the first pandemic wave: a crosssectional survey. Journal of Hospital Infection. 2021 Feb 1;108:168-73.

17. Mohamud Al, Mohamed SA, Abdullahi K. Assessments of a COVID-19 vaccine acceptance rate in population of Benadir region, Somalia.

18. Ahmed MA, Colebunders R, Gele AA, Farah AA, Osman S, Guled IA, Abdullahi AA, Hussein AM, Ali AM, Siewe Fodjo JN. COVID-19 Vaccine Acceptability and Adherence to Preventive Measures in Somalia: Results of an Online Survey. Vaccines. 2021 Jun;9(6):543.

\section{Tables}

Table:1. Age distribution

\begin{tabular}{|lll|}
\hline $\mathrm{N}$ & \multicolumn{1}{c|}{ Valid } & 210 \\
\cline { 2 - 3 } & Missing & 0 \\
\hline Mean & 28.23 \\
\hline Mode & 26 \\
\hline Std. Deviation & 2.920 \\
\hline
\end{tabular}




\begin{tabular}{|c|c|c|c|c|c|}
\hline Age & Vaccinated & Non-vaccinated & $\%$ Of Vaccinated & Total HCW & $P$ Value \\
\hline $18-27$ years & 13 & 100 & 11.5 & 113 & \\
\hline 28-35 years & 26 & 64 & 29 & 90 & $<0.01^{\star \star}$ \\
\hline $36-50$ years & 3 & 4 & 43 & 7 & \\
\hline \multicolumn{6}{|l|}{ Sex } \\
\hline Male & 31 & 87 & 26.2 & 118 & \\
\hline Female & 11 & 81 & 12 & 92 & $<0.01^{\star \star}$ \\
\hline \multicolumn{6}{|l|}{ Profession } \\
\hline Doctor & 31 & 71 & 30 & 102 & \\
\hline Nurse & 4 & 67 & 5.6 & 71 & \\
\hline \multirow[t]{2}{*}{ Radiology technician } & 2 & 12 & 14.2 & 14 & \\
\hline & & & & & $<0.182$ \\
\hline Anesthesia technician & 4 & 9 & 30.7 & 13 & \\
\hline Laboratory stuff & 1 & 9 & 10 & 10 & \\
\hline \multicolumn{6}{|l|}{ Total } \\
\hline & 42 & 168 & 20 & 210 & \\
\hline
\end{tabular}


Table:3. Reasons of not getting SARS-CoV-2 vaccine for unvaccinated participants

$\begin{array}{lllll}\text { Age } \quad \begin{array}{ll}\text { I did not get } \\ \text { the } \\ \text { opportunity }\end{array} & \begin{array}{l}\text { I do not think } \\ \text { it will be } \\ \text { beneficial to } \\ \text { me }\end{array} & \begin{array}{l}\text { I am afraid of } \\ \text { its } \\ \text { complications }\end{array} & \begin{array}{l}\text { I do not want to take } \\ \text { vaccine due to } \\ \text { religious or social } \\ \text { reasons }\end{array} & \text { No reason }\end{array}$

\begin{tabular}{lcccccccccc} 
& $\mathbf{n (})$ & $\%$ & $\mathbf{n ( )}$ & $\%$ & $\mathbf{n}()$ & $\%$ & $\mathbf{n}()$ & $\%$ & $\mathbf{n}()$ & $\%$ \\
\hline $\begin{array}{l}18-27 \\
\text { years }\end{array}$ & $(9)$ & 9 & $(20)$ & 20 & $(61)$ & 61 & $(3)$ & 3 & $(7)$ & 7 \\
\hline $\begin{array}{l}28-35 \\
\text { years }\end{array}$ & $(5)$ & 7.8 & $(18)$ & 28 & $(33)$ & 51.52 & $(0)$ & 0 & $(8)$ & 12.5 \\
\hline $\begin{array}{l}36-50 \\
\text { years }\end{array}$ & $(0)$ & 0 & $(1)$ & 25 & $(3)$ & 75 & $(0)$ & 0 & $(0)$ & 0
\end{tabular}

Sex

$\begin{array}{lllllllllll}\text { Male } & (7) & 8 & (22) & 25.2 & (46) & 53 & (1) & 1.1 & (11) & 12.6 \\ \text { Female } & (7) & 8.6 & (17) & 21 & (51) & 63 & (2) & 2.4 & (4) & 5\end{array}$

Profession

\begin{tabular}{lllllllllll} 
Doctor & $(8)$ & 11 & $(21)$ & 30 & $(34)$ & 48 & $(1)$ & 1 & $(7)$ & 10 \\
\hline Nurse & $(3)$ & 4.4 & $(13)$ & 19.4 & $(46)$ & 69 & $(0)$ & 0 & $(5)$ & 7.4 \\
\hline $\begin{array}{l}\text { Radiology } \\
\text { technician }\end{array}$ & $(1)$ & 8.3 & $(2)$ & 16.7 & $(5)$ & 41.6 & $(1)$ & 8.3 & $(3)$ & 25 \\
\hline $\begin{array}{l}\text { Anesthesia } \\
\text { technician }\end{array}$ & $(1)$ & 11.1 & $(1)$ & 11.1 & $(6)$ & 66.6 & $(1)$ & 11.1 & $(0)$ & 0 \\
\hline $\begin{array}{l}\text { Laboratory } \\
\text { stuff }\end{array}$ & $(1)$ & 11.1 & $(2)$ & 22.2 & $(6)$ & 66.6 & $(0)$ & 0 & $(0)$ & 0 \\
\hline Total & $(14)$ & 8.3 & $(39)$ & 23 & $(97)$ & 58 & $(3)$ & 1.7 & $(15)$ & 9 \\
\hline
\end{tabular}

$\mathrm{N}=$ sample size 
Table:4. This table summarizes the complications that vaccinated participants experienced regardless the type of vaccine

\begin{tabular}{lll} 
Variable & n Sample size & $\%$ \\
\hline Fever & 14 & 78 \\
\hline Body ache & 13 & 72.2 \\
\hline Pain at the site of injection & 8 & 44.4
\end{tabular}

Table:5. Correlations between SARS-CoV-2 vaccine and age, gender, and profession.

\begin{tabular}{llll} 
Variables & SARS-CoV-2 vaccine & \\
\cline { 2 - 4 } & Pearson correlation & Significant 2 tailed & N (sample size) \\
\hline Age & $-0.185^{\star \star}$ & $0.007^{\star *}$ & 210 \\
\hline Gender & $0.178^{\star \star}$ & 0.010 & 210 \\
\hline Profession & 0.092 & 0.182 & 210 \\
\hline
\end{tabular}

** indicates correlation between variables 